\title{
Random amplification of polymorphic DNA reveals clonal relationships among enteropathogenic Escherichia coli isolated from non-human primates and humans
}

V.M. Carvalho' K. Irino ${ }^{2}$, D. Onuma ${ }^{3}$ and A.F. Pestana de Castro ${ }^{3}$

\section{Correspondence}

V.M. Carvalho

Rua Dr. Bacelar, 1212

04026-002 São Paulo, SP

Brasil

Fax: +55-11-6204-6471

E-mail:vaniamc@uol.com.br

Research supported by FAPESP, CNPq and CAPES.

$\ldots \ldots \ldots \ldots \ldots \ldots \ldots$

Received May 23, 2006 Accepted September 25, 2006

\author{
${ }^{1}$ Faculdade de Medicina Veterinária, Instituto de Ciências da Saúde, \\ Universidade Paulista, São Paulo, SP, Brasil \\ ${ }^{2}$ Instituto Adolfo Lutz, São Paulo, SP, Brasil \\ ${ }^{3}$ Departamento de Microbiologia, Instituto de Ciências Biomédicas, \\ Universidade de São Paulo, São Paulo, SP, Brasil
}

\begin{abstract}
Enteropathogenic Escherichia coli (EPEC) strains are important agents of infantile diarrhea all over the world, gaining even greater importance in developing countries. EPEC have also been isolated from various animal species, but most isolates belong to serotypes that differ from those recovered from humans. However, it has been demonstrated that several isolates from non-human primates belong to the serogroups and/or serotypes related to those implicated in human disease. The objective of this study was to evaluate the genetic differences between thirteen strains isolated from non-human primates and the same number of strains isolated from human infections. Human isolates belonged to the same serogroup/serotype as the monkey strains and the evaluation was done by analysis of random amplified polymorphic DNA. Dendrogram analysis showed that there was no clustering between human and monkey strains. Human and non-human isolates of the EPEC serotypes O127:H40 and O128:H2 shared 90 and $87 \%$ of their bands, respectively, indicating strong genomic similarity between the strains, leading to the speculation that they may have arisen from the same pathogenic clone. To our knowledge, this study is the first one comparing genomic similarity between human and non-human primate strains and the results provide further evidence that monkey EPEC strains correlate with human EPEC, as suggested in a previous investigation.
\end{abstract}

Enteropathogenic Escherichia coli (EPEC) strains are defined by the ability to induce the lesion called "attaching and effacing" combined with the inability to produce Shiga toxins, in such a way that diarrhea is the result of pathogen-host membrane interactions $(1,2)$.
Key words - Escherichia coli

- Enteropathogenic Escherichia coli

- Attaching and effacing Escherichia coli

- Attaching and effacing

- Monkey

- Random amplified polymorphic DNA
EPEC strains are agents of infantile diarrhea all over the world, with even greater importance in developing countries, where the rate of infection and consequent infantile mortality reach higher levels $(1,3)$.

Attaching and effacing $E$. coli (AEEC) 
have been isolated from healthy and diarrheic animals of various species including pigs $(4,5)$, cows $(5,6)$, sheep and goats $(5,7)$, dogs and cats $(5,8)$, monkeys $(9,10)$, and rabbits $(11,12)$.

It has been recently demonstrated that several isolates from non-human primates belong to serogroups and/or serotypes related to those implicated in human disease, including the traditional EPEC serogroups O26, O127, O128, and O142. Carvalho et al. (9) speculated that the isolates from nonhuman primates may have the same origin as the human strains. Thus, the objective of the present study was to obtain complementary genetic evidence testing this assumption by evaluating genomic differences between strains isolated from humans and from nonhuman primates using the analysis of polymorphisms obtained by the random amplified polymorphic DNA (RAPD) technique.

Thirteen EPEC strains isolated from healthy and sick non-human primates, mostly marmosets, were compared with human strains belonging to the same serogroup/ serotype as the monkey isolates. Thrirteen human strains were obtained from children with diarrhea and selected from the culture collection of Instituto Adolfo Lutz, São Paulo,
SP, Brazil. The complete phenotypic and genotypic characterization of the monkey strains was described elsewhere $(9,13)$ and is summarized here in Table 1.

E. coli strains were grown in $2 \mathrm{~mL}$ brain heart infusion broth and incubated aerobically at $37^{\circ} \mathrm{C}$ overnight with shaking. DNA extraction was performed according to the recommendations of the Invitrogen-EasyDNA $^{\mathrm{TM}}$ kit, version E (Carlsbad, CA, USA). DNA concentration and purity were evaluated by electrophoresis on a $0.8 \%$ submersed agarose gel stained with ethidium bromide. The high DNA mass ladder (Invitrogen) was used as a molecular size marker.

RAPD-PCR was performed according to a protocol described elsewhere (14) using the primers OPR-04 (5'-CCCGTAGCAC3'), OPR-06 (5'-GTCTACGGCA-3') and OPR-08 (5'-CCCGTTGCCT3'). Each bacterial strain was analyzed in at least three independent reactions. PCR was carried out in an MJ Research model PTC-100 ${ }^{\mathrm{TM}}$ thermal cycler (Watertown, MA, USA). The RAPD products were visualized by electrophoresis on $1.4 \%$ agarose gels in $1 \mathrm{X}$ TBE (0.1 M Tris, $90 \mathrm{mM}$ boric acid, and $1 \mathrm{mM}$ EDTA, $\mathrm{pH}$ 8.3) stained with ethidium bromide. A 1-kb DNA ladder (Invitrogen) was

Table 1. Non-human primate EPEC strains isolated in the present study in Brazil.

\begin{tabular}{llccc}
\hline Strain No. & \multicolumn{1}{c}{ Species } & Clinical status & Serotype & bfpA gene/BFP expression \\
\hline 1 & Callithrix penicillata & HP & O128:H2 & $-/-$ \\
2 & Callithrix jacchus & HP & O127:H40 & $-/-$ \\
$3^{*}$ & Saguinus fuscicollis & SP & O127:H40 & $-/-$ \\
4 & Callithrix penicillata & HP & O33:H34 & $-/-$ \\
5 & Callithrix penicillata & HP & O167:H9 & $-/-$ \\
6 & Callithrix penicillata & SP & O167:H6 & +- \\
7 & Callithrix penicillata & SP & O142:H6 & $+/+$ \\
8 & Callithrix jacchus & HP & O132:H31 & $+/+$ \\
9 & Callithrix penicillata & HP & O132:H31 & $+/+$ \\
$10^{*}$ & Saguinus fuscicollis & SP & O26:H7 & $-/-$ \\
$11^{* *}$ & Callithrix penicillata & SP & O142:H6 & $+/+$ \\
$12^{* *}$ & Callithrix penicillata & SP & O142:H6 & $-/-$ \\
13 & Callithrix jacchus & SP & O142:H6 & $-/-$
\end{tabular}

${ }^{* * *}$ Strains recovered from the same animal. HP $=$ healthy non-human primate strain; SP = sick non-human primate strain. The $b f p A$ gene was detected by PCR and BFP expression was tested by Western blotting with BFP antiserum (9). 
used as a molecular size marker.

The RAPD-PCR profiles were recorded and analyzed with the NTSYS-pc program (NTSYS-ps, 1992-Numerical Taxonomical and Multivariate Analysis System, Exeter Software, Setauked, NY), version 1.7.

The RAPD technique has been used in the study of the clonal relationship between bacterial populations and is considered to be a useful tool for epidemiological studies $(15,16)$. The aim of the present study was to compare human and non-human primate strains isolated in São Paulo State. The use of the three primers generated 111 polymorphisms which were used in the construction of a binary matrix based on the presence or absence of shared bands. The dendrogram generated from the use of Dice coefficients is shown in Figure 1.

The results obtained showed that the distribution of human and monkey EPEC occurred uniformly among the two main clusters, $\mathrm{A}$ and $\mathrm{B}$, in contrast to the data reported by Venieri et al. (16), who demonstrated a difference between $E$. coli saprophyte strains isolated from animal and human sources.

Cluster B comprises all strains of sero- type $\mathrm{O} 26$ studied, independent of their origin, as well as serotypes O142:H6 and O132:H8 isolated from monkeys and humans, respectively. This group included strains recovered from sick individuals only.

Cluster A consisted of two other subgroups, A1 and A2. Subgroup A1 mainly consisted of isolates originating from healthy monkeys and subgroup A2 consisted predominantly of isolates originating from sick primates.

The monkey serotypes O128:H2, O127: $\mathrm{H} 40, \mathrm{O} 167: \mathrm{H} 9$, and O132:H31 were detected in cluster A1. The first two serogroups are widely referred to in the literature as the cause of diarrhea in children and the serotypes are prevalent in Brazil $(3,17)$. Due to their characterization regarding the presence and expression of $b f p A / \mathrm{BFP}$ and the intimin subtype, in a previous study by Blanco et al. (13) the monkey isolates of the present study were considered to be human serotypes.

The genomic profile determined by the RAPD technique in strains of serotypes O128:H2 and O127:H40 demonstrated a close correlation between the isolates from monkeys and humans, corroborating previ-

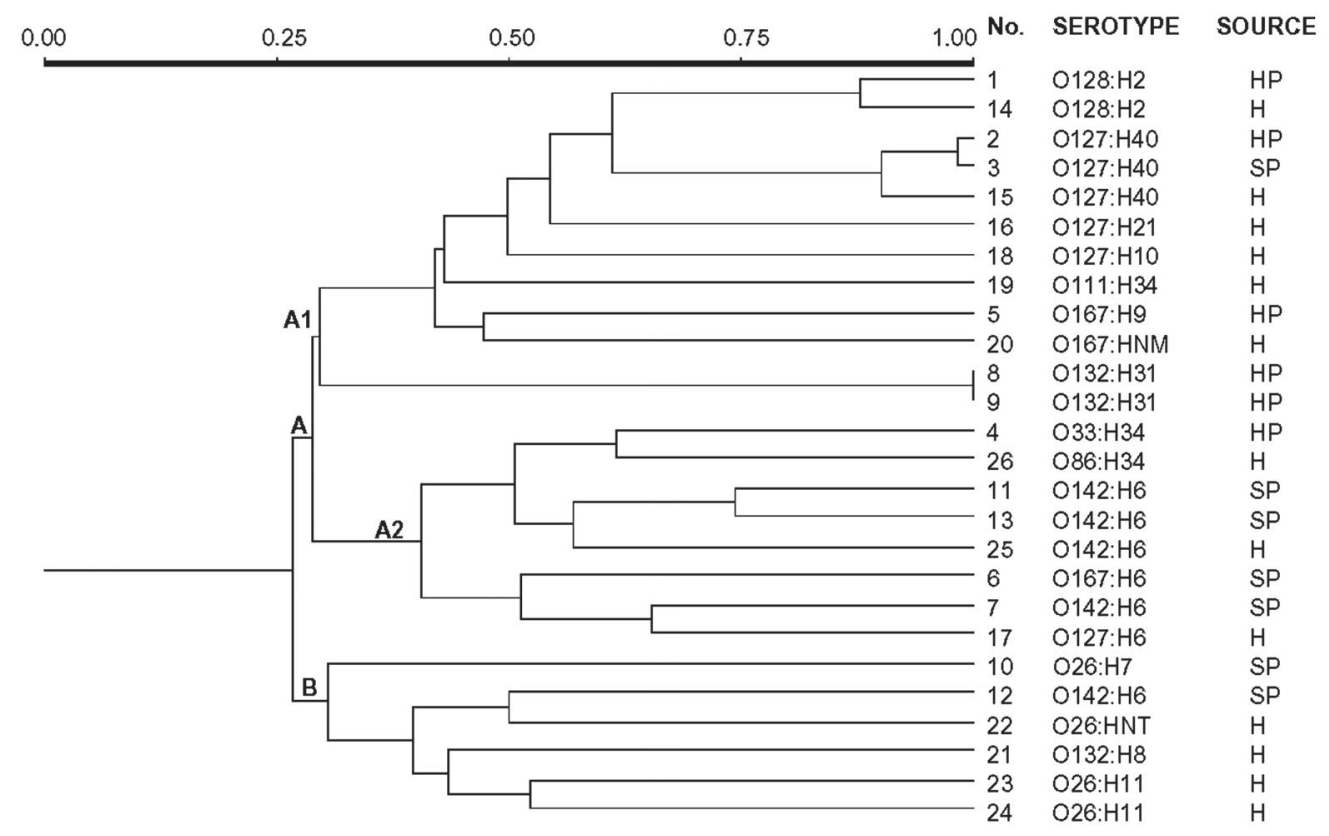

Figure 1. Dendrogram based on UPGMA cluster analysis of Dice coefficients. Clusters are indicated by the letters A, A1, A2 and $\mathrm{B} . \mathrm{HP}=$ healthy non-human primate strain; $\mathrm{SP}=$ sick nonhuman primate strain; $\mathrm{H}=\mathrm{hu}-$ man strain. 
ous results (9). Strains belonging to serotype O128:H2 shared $87 \%$ of the band profiles while strains belonging to serotype O127: H40 shared $90 \%$ of the bands, a fact leading to the speculation that these strains could have a common ancestor pathogen strain. On the other hand, strains of serotype O167:H9 presented approximately 50\% similarity to the human strain of the same serogroup. Although not included among the AEEC serotypes, O167:H9 was characterized as such in an outbreak involving a large number of children in Japan (18).

The two isolates of serotype O132:H31 were shown to be identical even though they were recovered from different individuals belonging to the same institution (data not shown), supporting the idea that potentially pathogenic clones may disseminate among individuals sharing the same environment. These O132:H31 strains had approximately $30 \%$ band identity with the remaining cluster strains. Both presented and expressed the $b f p A$ gene, showing a subtype of intimin $B$, LEE, inserted in phe $U$ and an FAS-positive reaction $(9,13)$. Despite showing human EPEC characteristics, the serotype is not included among human EPEC; however, serogroup O132 is related to infections in rabbits by REPEC (rabbit EPEC) in Brazil (12).

All isolates with the flagellar antigens H6 and H34 were included in cluster A2, with the majority of strains being recovered from sick monkeys. These results are similar to those obtained by other investigators who studied the genetic relationship between human EPEC serotypes $(17,19)$. The phenotypic and genotypic characteristics of the strains with these flagellar antigens allowed the authors to classify them as typical EPEC. In the present study, however, some of the isolates did not present the $b f p A$ gene (O33:H34 and O142:H6) nor did they express it in in vitro studies (O167:H6), despite their clonal relationship.

This is the first study comparing genomic similarity between human and non-human primate strains. The genomic similarity between serotypes O128:H2 and O127:H40, isolated from humans and monkeys, which are prevalent in Brazil in children with diarrhea $(3,17)$, supports the previously raised hypothesis that monkeys carry human EPEC strains $(9,13)$. These serotypes are classified as atypical EPEC and have been considered to be emergent human pathogens in developing countries (3).

Non-human primates are important models for the study of human disease, due to their phylogenetic proximity and similar susceptibility to human pathogens (20). The verification that these animals carry human EPEC strains opens the possibility of using these primates as models to study these infections, since Vallance and Finlay (2) emphasized the need to study the pathogenesis of EPEC in models genetically and immunologically more closely related to humans.

\section{References}

1. Nataro JP, Kaper JB. Diarrheagenic Escherichia coli. Clin Microbiol Rev 1998; 11: 142-201.

2. Vallance BA, Finlay BB. Exploitation of host cells by enteropathogenic Escherichia coli. Proc Natl Acad Sci U S A 2000; 97: 87998806.

3. Trabulsi LR, Keller R, Tardelli Gomes TA. Typical and atypical enteropathogenic Escherichia coli. Emerg Infect Dis 2002; 8: 508513.

4. Martins MF, Martinez-Rossi NM, Ferreira A, Brocchi M, Yano T, Castro AF, et al. Pathogenic characteristics of Escherichia coli strains isolated from newborn piglets with diarrhea in Brazil. Vet Microbiol 2000; 76: 51-59.

5. Krasue G, Zimmerman S, Beutin L. Investigation of domestic animals and pets as a reservoir for intimin-(eae) gene positive Escherichia coli types. Vet Microbiol 2005; 106: 1290-1298.

6. Aidar L, Penteado AS, Trabulsi LR, Blanco JE, Blanco M, Blanco J, et al. Subtypes of intimin among non-toxigenic Escherichia coli from diarrheic calves in Brazil. Can J Vet Res 2000; 64: 15-20.

7. Cid D, Ruiz-Santa-Quiteria JA, Marin I, Sanz R, Orden JA, Amils R, et al. Association between intimin (eae) and EspB gene subtypes in 
attaching and effacing Escherichia coli strains isolated from diarrhoeic lambs and goat kids. Microbiology 2001; 147: 2341-2353.

8. Nakazato G, Gyles C, Ziebell K, Keller R, Trabulsi LR, Gomes TA, et al. Attaching and effacing Escherichia coli isolated from dogs in Brazil: characteristics and serotypic relationship to human enteropathogenic E. coli (EPEC). Vet Microbiol 2004; 101: 269-277.

9. Carvalho VM, Gyles CL, Ziebell K, Ribeiro MA, Catao-Dias JL, Sinhorini IL, et al. Characterization of monkey enteropathogenic Escherichia coli (EPEC) and human typical and atypical EPEC serotype isolates from Neotropical nonhuman primates. J Clin Microbiol 2003; 41: 1225-1234.

10. Thomson JA, Scheffler JJ. Hemorrhagic typhlocolitis associated with attaching and effacing Escherichia coli in common marmosets. Lab Anim Sci 1996; 46: 275-279.

11. Milon A, Oswald E, De Rycke J. Rabbit EPEC: a model for the study of enteropathogenic Escherichia coli. Vet Res 1999; 30: 203-219.

12. Penteado AS, Ugrinovich LA, Blanco J, Blanco M, Blanco JE, Mora $A$, et al. Serobiotypes and virulence genes of Escherichia coli strains isolated from diarrheic and healthy rabbits in Brazil. Vet Microbiol 2002; 89: 41-51.

13. Blanco M, Blanco JE, Blanco J, Carvalho VM, Onuma DL, Pestana de Castro AF. Typing of intimin (eae) genes in attaching and effacing Escherichia coli strains from monkeys. J Clin Microbiol 2004; 42 : 1382-1383.
14. Peixoto JC, Bando SY, Ordonez JA, Botelho BA, Trabulsi LR, Moreira-Filho CA. Genetic differences between Escherichia coli 026 strains isolated in Brazil and in other countries. FEMS Microbiol Lett 2001; 196: 239-244.

15. Girao DM, Bando SY, Girao VBC, Moreira-Filho CA, Fracalanzza SEL, Trabulsi LR, et al. Characterization of typical and atypical enteropathogenic Escherichia coli (EPEC) strains of the classical O55 serogroup by RAPD analysis. Rev Microbiol 1999; 30: 365368.

16. Venieri D, Vantarakis A, Komninou G, Papapetropoulou M. Differentiation of faecal Escherichia coli from human and animal sources by random amplified polymorphic DNA-PCR (RAPD-PCR). Water Sci Technol 2004; 50: 193-198.

17. Campos LC, Franzolin MR, Trabulsi LR. Diarrheagenic Escherichia coli categories among the traditional enteropathogenic E. coli O serogroups - a review. Mem Inst Oswaldo Cruz 2004; 99: 545-552.

18. Ohtani Y, Kameda M, Tamura K. An outbreak of gastroenteritis possibly caused by Escherichia coli 0167:H9. Kansenshogaku Zasshi 1991; 65: 35-39.

19. Whittam TS, McGraw EA. Clonal analysis of EPEC serogroups. Rev Microbiol 1996; 27: 7-16.

20. Wolfe ND, Escalante AA, Karesh WB, Kilbourn A, Spielman A, Lal AA. Wild primate populations in emerging infectious disease research: the missing link? Emerg Infect Dis 1998; 4: 149-158. 